\title{
Enhanced Visualization of the Cervical Vertebra during Intraoperative Fluoroscopy Using a Shoulder Traction Device
}

\author{
Van Tri Truong ${ }^{1}$, Fidaa Al-Shakfa ${ }^{1}$, Ghassan Boubez ${ }^{1}$, Daniel Shedid ${ }^{2}$, Sung-Joo Yuh ${ }^{2}$, Zhi Wang ${ }^{1}$ \\ ${ }^{1}$ Division of Orthopedics, Centre Hospitalier de l'Université de Montréal (CHUM), University of Montreal, Montreal, QC, Canada \\ ${ }^{2}$ Division of Neurosurgery, Centre Hospitalier de l'Université de Montréal (CHUM), University of Montreal, Montreal, QC, Canada
}

Study Design: A retrospective, matched cohort study of a prospective database.

Purpose: To evaluate the efficacy and safety of the Cervision system (Spinologics, Montreal, Canada), a new shoulder traction device that improves the fluoroscopic visualization of the lower cervical spine using caudal traction of the shoulders out of the radiographic field.

Overview of Literature: Operating at a wrong level is a common error that may be committed by nearly $50 \%$ of surgeons during their career. Intraoperative fluoroscopy of the cervical vertebrae is an extremely important step in cervical spine surgery. Optimal lateral cervical radiography of the C1-T1 vertebrae is not always possible due to overlap of the shoulders.

Methods: In this study, a group of patients ( $n=33$, device group) underwent surgery with the new device used to apply caudal traction to both shoulders, and another group of patients ( $\mathrm{n}=33$, matched control group) had surgery with the tape traction. Data about the lowest vertebra visible on lateral fluoroscopic view, installation time, skin irritation under the traction area, and postoperative brachial palsy were recorded, and these parameters were analyzed using the $t$-test.

Results: The mean numbers of visible cervical vertebra were $6.3 \pm 0.41$ in the device group and $5.6 \pm 0.32$ in the matched control group $(p<0.01$, unpaired $t$-test). The mean installation times were $83.9 \pm 5.15$ minutes in the device group and $73.7 \pm 6.32$ minutes in the matched control group $(p<0.02)$. Seven patients from the matched control group presented with skin irritation. However, none of the patients from the device group had the condition ( $p=0.005$, Pearson chi-square test). Postoperative brachial palsy was not observed in both groups.

Conclusions: The Cervision system is more effective and superior to tape traction in pulling the shoulders down to improve the visualization of the cervical vertebra on lateral fluoroscopic view during cervical spine surgery.

Keywords: Shoulder; Cervical; Spine; Surgery; Fluoroscopy

Received Aug 7, 2019; Revised Sep 18, 2019; Accepted Oct 2, 2019

Corresponding author: Van Tri Truong

Division of Orthopedics, Centre Hospitalier de l'Université de Montréal (CHUM), University of Montreal, 1051 Sanguinet Street, Montreal, QC H2X 3E4 Canada

Tel: +1-438-334-3141, Fax: +1-514-412-7619, E-mail: drtruongtri@gmail.com

*The portion of this work was presented at the Oral Podium in Canadian Spine Society-2019 Scientific Conference, Toronto, Canada, February 28, 2019. 


\section{Introduction}

Intraoperative fluoroscopy of the cervical vertebrae is a critical step in preventing wrong-level spine surgery. In the evaluation of implants and estimation of the cervical curvature [1-3], operating at a wrong level is a common error that may be committed by nearly $50 \%$ of surgeons during their career $[4,5]$. Optimal lateral cervical radiography of the $\mathrm{C} 1-\mathrm{T} 1$ vertebrae is not always possible due to overlap of the shoulders. Adhesive tape is frequently used for the caudal traction of a patient's shoulders to improve imaging. However, the tape may hinder vascular access, limit the use of monitoring equipment on the arms, and cause skin irritation. In this study, the efficacy and safety of the Cervision system, which is a new devised used to improve the fluoroscopic visualization of the lower cervical spine via caudal retraction of a patient's shoulders, was assessed.

\section{Materials and Methods}

\section{Device}

The Cervision system (Spinologics, Montreal, Canada) comprised two sleeves, each wrapped around the patient's arm and connected to a strap on the same side (Fig. 1). Physicians can easily wrap or unwrap these sleeves as needed for vascular access and monitoring. Various caudal tractions on the shoulders can be achieved using a
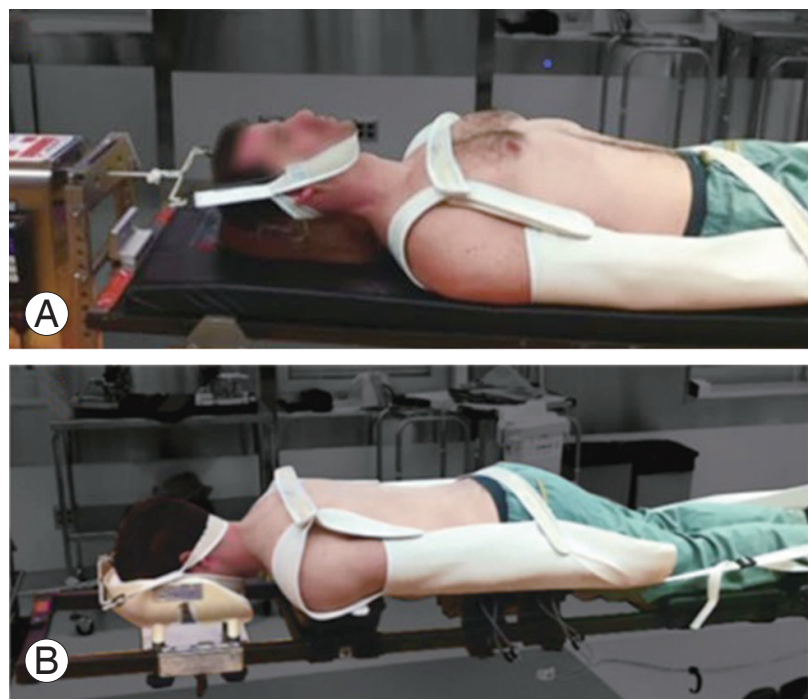

Fig. 1. The Cervision system (Spinologics, Montreal, Canada) with two sleeves covering the arms connected to two straps enclosing the shoulders while the patients are in prone position (A) and supine position (B). tensioner (Fig. 2), which facilitates relaxation when critical imaging is completed. This system is fully adjustable in all patients.

\section{Study population}

A retrospective, matched cohort study of a prospective database was conducted to evaluate the efficacy of the Cervision system (Spinologics). This study was approved by the Research Ethics Committee of Centre Hospitalier de l'Université de Montréal (approval no., 18.182). Informed consent was obtained from all individual participants included in the study. The data of 33 consecutive patients who underwent cervical spine surgery with the Cervision system were used in this study, and the operation was conducted by a senior surgeon at a single institution during a 5-year period from October 23, 2013 to October 25, 2018. The matched control group included 33 consecutive patients who underwent surgery conducted by other spine surgeons at the same institution during the same period. In this group, adhesive tape traction was used to provide caudal traction on the shoulders of patients.

Personal data, age, body mass index, surgical intervention, number of visible cervical vertebrae on intraoperative fluoroscopy, installation time, and perioperative complications of each patient were recorded. Installation time was calculated from the beginning of anesthesia induction to the time of skin incision.

\section{Intraoperative procedure}

All patients underwent surgery under general anesthesia, and the patient's head was placed on a foam headrest

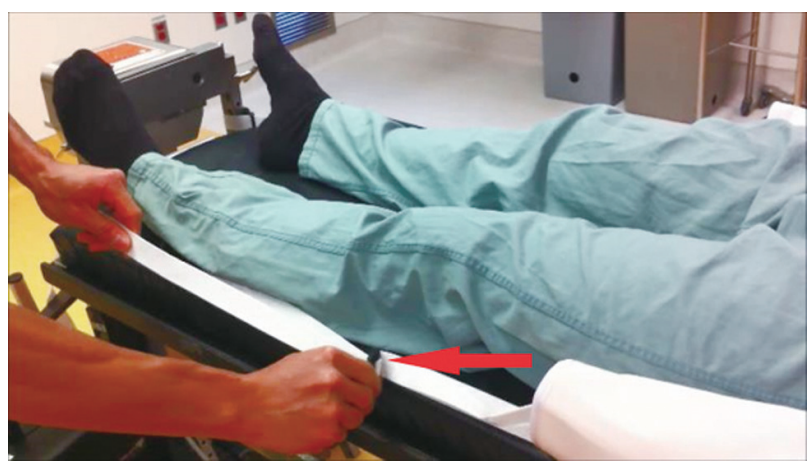

Fig. 2. The Cervision system (Spinologics, Montreal, Canada) has adjustable locks (arrow) that are used to reduce the tension of shoulder traction as needed. 
while in supine position or in the Mayfield frame in prone position. The Cervision system, or adhesive tape, was applied by the surgical team to pull the patient's shoulders caudally. After the surgical field was sterilized and draped, lateral fluoroscopy from the $\mathrm{C} 1$ vertebra to the lowest visible cervical vertebrae was conducted with a mobile Carm machine.

The lowest vertebral body visible was identified on the partially overlapping image of the shoulders on lateral projection fluoroscopy. The superior body endplate was clearly visible. However, the inferior endplate was obscured.

At the end of surgery, skin irritation under the traction area was evaluated and classified using a visual grading scale. The segmental strength of the upper extremities was assessed on the first postoperative day.

\section{Statistical analysis}

Descriptive statistics (mean \pm standard deviation) were used to analyze continuous variables, including the number of visible vertebrae and installation time. Student $t$-test was utilized to compare differences between the variables in the two cohorts.

\section{Results}

The collected data are summarized in Table 1. Of the 33 patients who had surgery using the Cervision cohort, 18 (54.6\%) underwent anterior approach surgery for cervical discectomy and fusion, and $15(45.4 \%)$ had posterior approach surgery for multilevel cervical laminectomy and fusion. In the matched control group, the proportion of surgical approaches was similar.

In patients undergoing anterior cervical spine surgery, a significant difference was observed in terms of cervical vertebra visualization on the lateral radiographic image between the two cohorts ( $p=0.03, t$-test). The number of visible cervical vertebra was higher in the subgroup (about $15 \%$ of patients) who underwent posterior approach surgery than in the matched control group. However, the

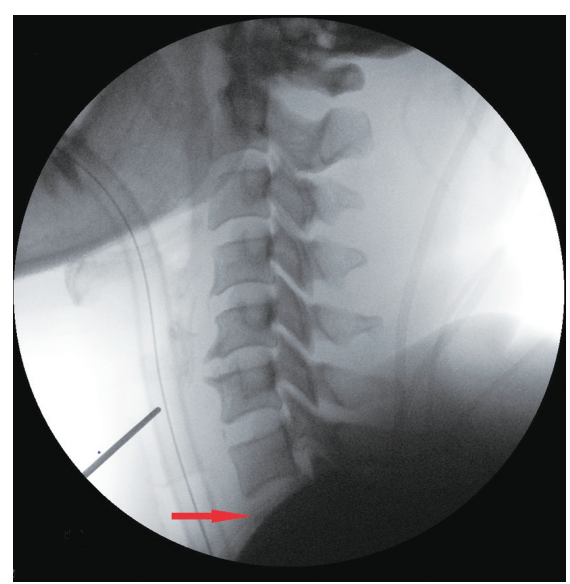

Fig. 3. Intraoperative lateral fluoroscopy showing the T1 vertebrae (arrow).

Table 1. Summary data of 2 cohorts

\begin{tabular}{|c|c|c|c|}
\hline Characteristic & Cervision cohort & Control cohort & $p$-value \\
\hline No. of patients & 33 & 33 & \\
\hline Age (mean yr) & 56.8 & 59.7 & \\
\hline Anterior cervical discectomy and fusion (case) & 18 & 18 & \\
\hline $\mathrm{BMI}\left(\mathrm{kg} / \mathrm{m}^{2}\right)$ & $30.96 \pm 3.49$ & $26.93 \pm 2.94$ & 0.10 \\
\hline Mean no. of cervical vertebra visualized on lateral fluoroscopy & $6.67 \pm 0.91$ & $6.06 \pm 0.72$ & 0.03 \\
\hline Multilevel cervical laminectomy and fusion (case) & 15 & 15 & \\
\hline $\mathrm{BMI}\left(\mathrm{kg} / \mathrm{m}^{2}\right)$ & $31.21 \pm 4.50$ & $25.52 \pm 3.04$ & 0.5 \\
\hline Mean no. of cervical vertebra visualized on lateral fluoroscopy & $5.93 \pm 1.39$ & $5.07 \pm 0.88$ & 0.05 \\
\hline T1 vertebrae visualized on lateral fluoroscopy (case) & 5 & 0 & 0.02 \\
\hline Installation time (min) & $83.9 \pm 5.2$ & $73.7 \pm 6.3$ & $<0.02$ \\
\hline Skin irritation grade I (case) & 0 & 6 & $<0.01$ \\
\hline Skin irritation grade II (case) & 0 & 1 & \\
\hline Postoperative brachial palsy (case) & 0 & 0 & \\
\hline
\end{tabular}

Values are presented as number or mean \pm standard deviation. Bold type is considered statistically significant. $\mathrm{BMI}$, body mass index. 


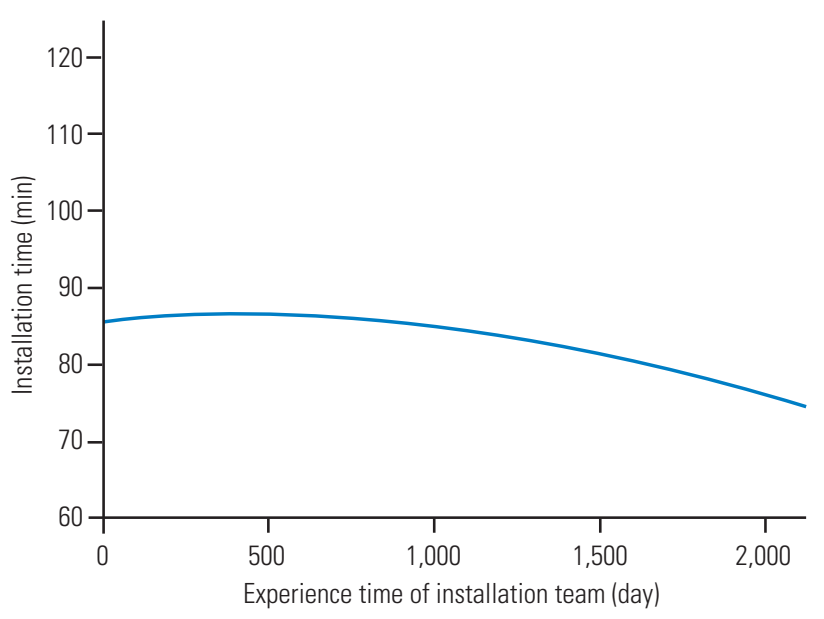

Fig. 4. Learning curve for the installation time of the Cervision system (Spinologics, Montreal, Canada).

difference was not significantly different $(p=0.05)$. Five patients (15.2\%) presented with visible T1 vertebra on fluoroscopy in the Cervision cohort group (Fig. 3). However, none of the patients in the matched control group presented with such condition ( $p=0.02$, Pearson chi-square test).

The installation time was longer by about 10 minutes in the Cervision group than in the matched control group $(p<0.02)$, and analysis of the learning curve with the Cervision system showed improvement over time (Fig. 4).

In the matched control group, six patients had grade 1 skin irritation under the traction area, and one patient had grade II skin irritation. Meanwhile, none of the patients in the Cervision group had skin irritation. The result was significantly different between the two groups $(p<0.01$, Pearson chi-square test).

Moreover, none of the patients in the two groups presented with postoperative brachial palsy.

\section{Discussion}

Radiographic visualization of the cervical spine during cervical spine surgery is essential and performed routinely by most spine surgeons [6,7]. Optimal cervical spine fluoroscopy can show the $\mathrm{C} 1-\mathrm{T} 1$ vertebrae on lateral projection. However, visualization is not always possible because the patient's shoulders may obscure the lower cervical vertebrae. Varying techniques have been proposed to increase the visualization of the lower cervical vertebrae during spine surgery. Modified oblique cross-table fluoroscopy, which was proposed by Singh et al. [8], may help obtain an image obscured by the shoulders. However, images obtained using this technique are not clear and may cause uncertainty with regard to the vertebral level. Caudal displacement of the patients' shoulders during fluoroscopic examination facilitates better imaging of the cervical spine, thereby reducing the duration of intraoperative fluoroscopy and total radiation exposure. An assistant may pull the patient's wrists distally during fluoroscopy. However, the assistant may be exposed to increased radiation, and wrist traction may displace the sterile drapes and intravenous catheters. Some surgeons attach adhesive tapes to the arms and shoulders, which may cause skin irritation and interfere with catheters and monitoring equipment. Moreover, continuous traction throughout the surgical procedure is not necessary and will likely increase the risk of brachial plexus palsy. In 2013, Witiw et al. [9] has proposed the use of a device called the Citow Cervical Visualizer, which is applied to the patient's acromion as needed. Moreover, Marangoz has reported an intraoperative traction device that is used to pull the shoulders [10]. However, with the utilization of both devices, the user is exposed to increased radiation due to positioning $[9,10]$. Moreover, whether these two devices are more effective than tape traction in visualizing the lower cervical vertebrae on fluoroscopy is not elucidated.

The Cervision system is designed to improve the visualization of the cervical vertebrae during cervical spine surgery. The present study showed that a significantly higher number of cervical vertebrae was visualized using the Cervision system in the subgroup who underwent anterior approach surgery than in the matched control group. In patients who underwent posterior cervical spine surgery, the device may be superior to tape traction. However, the results did not significantly differ. The analysis of data showed that the matched control group was at higher risk of skin irritation under the traction area than the device group. Postoperative brachial palsy was not observed in both groups. The device was compatible with both prone and supine positions and was effective in patients of all sizes both in the operating room and radiology department. Intravenous lines and monitoring devices in the patient's arms were protected by the sleeves. Moreover, the force of the shoulder traction can be adjusted during surgery to prevent excess brachial plexus traction. The installation time of the new device was longer than that of the taping procedure, and analysis of the learning curve showed improvement over time. 
The present study had several limitations. Two groups of patients were included, and the number of participants in each subgroup was low. Thus, a randomized controlled trial should be conducted as it provides better evidence on the efficacy of the Cervision system versus the commonly used tape traction during cervical spine surgery.

\section{Conclusions}

The Cervision system is more effective and superior to tape traction in producing caudal shoulder displacement to increase the visualization of the lower cervical vertebra levels during supine intraoperative fluoroscopy.

\section{Conflict of Interest}

No potential conflict of interest relevant to this article was reported.

\section{Author Contributions}

Conception and design: Zhi Wang, Van Tri Truong; administrative support: Zhi Wang; provision of study materials or patients: Ghassan Boubez, Daniel Shedid, SungJoo Yuh, Zhi Wang; collection and assembly of data: Van Tri Truong, Fidaa Al-Shakfa; data analysis and interpretation: Fidaa Al-Shakfa, Van Tri Truong; and manuscript writing: all authors

\section{References}

1. Yoshimoto H, Sato S, Hyakumachi T, Yanagibashi Y, Kanno T, Masuda T. Clinical accuracy of cervical pedicle screw insertion using lateral fluoroscopy: a radiographic analysis of the learning curve. Eur Spine J 2009;18:1326-34.
2. Ito Y, Sugimoto Y, Tomioka M, Hasegawa Y, Nakago $\mathrm{K}$, Yagata Y. Clinical accuracy of 3D fluoroscopyassisted cervical pedicle screw insertion. J Neurosurg Spine 2008;9:450-3.

3. Morgan $\mathrm{H}$. Wrong disc space level surgery: medicolegal implications. Surg Neurol 2004;62:278.

4. Mody MG, Nourbakhsh A, Stahl DL, Gibbs M, Alfawareh M, Garges KJ. The prevalence of wrong level surgery among spine surgeons. Spine (Phila Pa 1976) 2008;33:194-8.

5. Goodkin R, Laska LL. Wrong disc space level surgery: medicolegal implications. Surg Neurol 2004;61:323-42.

6. Cha SH, Kim C, Choi BK, Kim HJ, Baek SY. C-arm assessment of cervical pedicle screw: screw coaxial fluoroscopy and oblique view. Spine (Phila Pa 1976) 2007;32:1721-7.

7. Ondra SL, Marzouk S, Ganju A, Morrison T, Koski T. Safety and efficacy of $\mathrm{C} 2$ pedicle screws placed with anatomic and lateral C-arm guidance. Spine (Phila Pa 1976) 2006;31:E263-7.

8. Singh H, Meyer SA, Hecht AC, Jenkins AL 3rd. Novel fluoroscopic technique for localization at cervicothoracic levels. J Spinal Disord Tech 2009;22:615-8.

9. Witiw CD, Citow JS, Ginsberg HJ, et al. A novel device to simplify intraoperative radiographic visualization of the cervical spine by producing transient caudal shoulder displacement: a 2-center case series of 80 patients. J Neurosurg Spine 2013;19:697-700.

10. Marangoz A, Cokluk C, Kuruoglu E, Aydin K, Altun A. Shoulder traction device for enhanced conventional fluoroscopy during cervical spine operations. Neurol Med Chir (Tokyo) 2013;53:82-4. 